\title{
La fuerza explicativa de los métodos mixtos. Una ejemplificación a partir de las diferencias territoriales en la emancipación familiar en Catalunya*
}

\section{Joan Miquel Verd i Pericàs}

Universitat Autònoma de Barcelona. Departament de Sociologia

Centre d'Estudis Sociològics sobre la Vida Quotidiana i el Treball (QUIT)

08193 Bellaterra (Barcelona). Spain

joanmiquel.verd@uab.cat

\section{Resumen}

El artículo aborda las diferencias territoriales en la emancipación juvenil en Catalunya utilizando una combinación de técnicas cuantitativas y cualitativas. Esta aproximación metodológica permite realizar la evaluación de la capacidad explicativa de diversos modelos teóricos. Los resultados obtenidos muestran que las diferencias territoriales son el resultado de una combinación de factores múltiples, que normalmente no se consideran de forma conjunta en las investigaciones empíricas. Como conclusión, se defiende que es precisamente la mezcla de datos cuantitativos y cualitativos la que hace posible abordar conjuntamente factores provenientes de diferentes aproximaciones teóricas y relacionados con diferentes niveles de la realidad social.

Palabras clave: emancipación juvenil, articulación metodológica, métodos mixtos, Catalunya.

Abstract. The explicative strength of mixed methods. The example of territorial differences in family emancipation in Catalonia

The article addresses the territorial differences of youth emancipation in Catalonia by using a combination of quantitative and qualitative methods. This methodological approach allows the evaluation of the explanatory capacity of several theoretical models. The results obtained show that the differences among territories are the result of a combination of multiple factors that usually are not jointly considered in empirical research. To conclude, it is stated that it is just the mixing of quantitative and qualitative data that makes possible addressing jointly factors stemming from different theoretical approaches and related to different levels of social reality.

Key words: youth emancipation, methodological articulation, mixed methods, Catalonia.

* Quisiera agradecer a Pedro López el trabajo de recodificación realizado con las variables del censo de 2001. También agradezco los comentarios y las observaciones realizados por parte de los evaluadores anónimos, que han contribuido a mejorar el artículo. 


\section{Sumario}

Introducción

1. Diseño de la investigación

2. Diferencias en la emancipación familiar juvenil por grupos de edad y ámbitos territoriales

3. El peso de los factores económicos y laborales

4. La prolongación de los estudios como factor explicativo
5. Las estrategias familiares y la clase social de origen

6. Los factores culturales y las expectativas en la emancipación

7. Conclusiones

Bibliografía

\section{Introducción}

A pesar de la continua actualidad de la problemática relacionada con la emancipación juvenil y las dificultades de acceso a la vivienda, las investigaciones que han abordado empíricamente esta cuestión no son numerosas. En lugar de investigaciones rigurosas centradas en la exploración de todos los posibles factores explicativos, son más frecuentes los planteamientos parciales y reduccionistas presentes en la prensa o en el debate público.

Muy a menudo, además, la información considerada es únicamente cuantitativa, centrada en las barreras de carácter económico a la emancipación (Vergés, 1997). Ello implica dejar de lado posibles factores explicativos que son difícilmente abordables de forma cuantitativa, con la consecuencia de suponer, ya desde el planteamiento inicial, una visión limitada de la problemática. Como es sabido, las técnicas cualitativas, al ofrecer información de mayor riqueza y profundidad, permiten realizar un análisis detallado de los marcos de referencia, las representaciones y las estrategias de las personas, factores que son centrales en algunas de las explicaciones que abordan la emancipación juvenil. Por otra parte, es necesario reconocer que un diseño exclusivamente cualitativo también plantea dificultades importantes, puesto que puede suponer sobredimensionar el peso de los factores culturales o relacionados con los imaginarios de los jóvenes, dejando de lado las condiciones materiales de su existencia.

Intentando salvar la profunda división metodológica que a menudo separa los dos tipos de planteamientos que acabamos de señalar, el presente artículo apuesta por el estudio de la emancipación juvenil con una aproximación metodológica de carácter mixto o multiestratégico (Bericat, 1998; Lozares, Martín y López, 1998; Domínguez y Coco, 2000; Tashakkori y Teddlie, 2003; Creswell y Plano-Clark, 2007). El artículo pretende mostrar, además, que este tipo de aproximación ofrece dos ventajas principales: en primer lugar, permite considerar el máximo de factores explicativos, puesto que no deja de lado ni variables cuantitativas ni variables cualitativas, permitiendo además identificar la articulación causal de los diferentes factores; en segundo lugar, y como consecuencia de lo anterior, posibilita contrastar empíricamente una pluralidad 
de modelos teóricos, tanto los que inciden en los elementos más materiales y «objetivos» (generalmente operativizados y medidos utilizando técnicas cuantitativas), como los que inciden en los elementos más culturales y «subjetivos» (generalmente operativizados y medidos utilizando técnicas cualitativas).

Aunque existen trabajos empíricos en que se afirma que los factores que inciden en las posiciones y en las expectativas de la juventud con respecto a su emancipación son múltiples — como los de Casal y otros (2004), Trilla y López (2005) o Fernández, Batalla y Micó (2005), por ejemplo-, raramente se acomete la tarea de explicitar cuál es la imbricación o la articulación de esta pluralidad de factores. En este sentido, tal y como destaca Elliott (2005), el uso combinado de información cuantitativa y cualitativa permite realizar una buena aproximación causal, puesto que las explicaciones resultantes «no sólo describen regularidades en el modo en que unas variables se comportan respeto a las otras, sino que también ofrecen un posible mecanismo subyacente a la relación» (2005: 173) $)^{1}$.

Con el objetivo de mostrar en qué medida se pueden sostener las afirmaciones anteriores, en los capítulos siguientes se analizarán las diferencias territoriales en Catalunya en lo que hemos denominado emancipación familiar, entendida como la situación en que un joven o una joven reside en una vivienda del que él/ella o su pareja son la persona principal de referencia. Se comprobará, en definitiva, hasta qué punto el uso combinado de información cuantitativa y cualitativa permite explicar la diversidad territorial observada, así como cuáles de los factores apuntados por los diferentes modelos explicativos existentes encajan mejor con la realidad descrita ${ }^{2}$. Los datos utilizados fueron obtenidos en el marco del proyecto La realitat juvenil a Catalunya ${ }^{3}$ (López, Valls, Verd y Vidal, 2006), cuyos ámbitos de interés eran considerablemente amplios (educación, trabajo, vivienda, cultura y estilos de vida, salud, participación, y cohesión social y equilibrio territorial), y, por lo tanto, constituyen sólo una pequeña parte de la información recogida durante la investigación. Asimismo, la explotación realizada para este artículo supone un análisis más profundo del llevado a cabo inicialmente en el proyecto.

1. Esta afirmación puede entenderse mejor si se tiene en cuenta que las técnicas cuantitativas y cualitativas ofrecen información de distinto tipo, de carácter intensivo en el primer caso y de carácter extensivo en el segundo.

2. La evaluación de este «ajuste» se hará siguiendo una estrategia comparativa alejada de los análisis cross-section, forzosamente la única vía posible si se quiere integrar información de carácter no estadístico. Véase Lallement (2003), para una interesante discusión alrededor de esta cuestión.

3. El estudio fue encargado por el Observatori Català de la Juventut, de la Secretaría General de Juventud de la Generalitat de Catalunya al Centre d'Estudis Sociològics sobre la Vida Quotidiana i el Treball, de la Universidad Autónoma de Barcelona y al Observatori del Tercer Sector, y se desarrolló entre diciembre de 2005 y marzo de 2006. En él participaron, por orden alfabético: Marina Albinyana, Jonatan Castaño, Clara Créixams, Clèlia Colombo, Anna Font, Pedro López, Bego Martínez, Sergio Porcel, Núria Quintana, Gerard Quiñones, Maria Sureda, Daniel Tarragó, Anna Torrent, Núria Valls, Joan Miquel Verd, Pau Vidal y Anna Vilarrasa. 
El artículo se estructura presentando, en primer lugar (punto 1), el diseño metodológico aplicado y las fuentes de datos utilizadas, a continuación (punto 2) se muestran los valores de la emancipación familiar en Catalunya desglosados por ámbitos territoriales. El análisis de las disparidades que esta comparación presenta para el grupo de edad de entre 25 y 34 años (que es el periodo en que se concentran los procesos emancipatorios de los jóvenes y las jóvenes) constituye el grueso del artículo. En los puntos 3, 4, 5 y 6 se evalúan, por este orden, las explicaciones basadas en factores económicos y laborales, las basadas en el alargamiento de la formación, las basadas en las estrategias familiares y, finalmente, las basadas en factores culturales. La consideración conjunta de información cuantitativa y cualitativa apunta hacia la relevancia de los razonamientos basados en estos dos últimos modelos en la explicación de las diferencias territoriales detectadas. El artículo termina con un apartado de conclusiones, que pone de manifiesto la ausencia de monocausalidad y la necesidad de realizar planteamientos de investigación que combinen técnicas cuantitativas y cualitativas.

\section{Diseño de la investigación}

Como se ha indicado anteriormente, los datos que se analizan en el artículo tienen su origen en el proyecto La realitat juvenil a Catalunya (López, Valls, Verd y Vidal, 2006). La investigación ha tenido la particularidad de centrarse en las características sociales de las diferentes comarcas y ámbitos territoriales en que puede dividirse el Principado y ha combinado datos de carácter estadístico (estadística de población de 1996 y censo de 2001) con información cualitativa proveniente de treinta y cinco grupos de discusión con jóvenes realizados por todo el territorio catalán. La atención prestada al nivel territorial ha dotado de un fuerte carácter comparativo a la investigación, y ha permitido poner de relieve informaciones que, de otro modo, quedan diluidas en una «uniformidad» catalana marcada profundamente por el peso demográfico de Barcelona y su región metropolitana. En el presente artículo, se toman como unidades de análisis los ámbitos territoriales (àmbits territorials), que son agrupaciones de comarcas enteras que suponen una división administrativa territorial más homogénea que la planteada por las provincias. Los ámbitos territoriales considerados son los habituales: Alt Pirineu i Aran, Àmbit Metropolità, Camp de Tarragona, Comarques Centrals, Comarques Gironines, Àmbit de Ponent y Terres de l'Ebre. En la figura 1 se muestran gráficamente estas agrupaciones ${ }^{4}$.

El tipo de diseño llevado a cabo en la investigación —utilizaré aquí la terminología de Creswell (Creswell, 2003; Creswell y Plano-Clark, 2007)— ha sido el resultado de la integración de un diseño secuencial con un diseño concu-

4. En López, Valls, Verd y Vidal (2006) se ofrece el listado completo de comarcas que corresponden a cada ámbito territorial. 


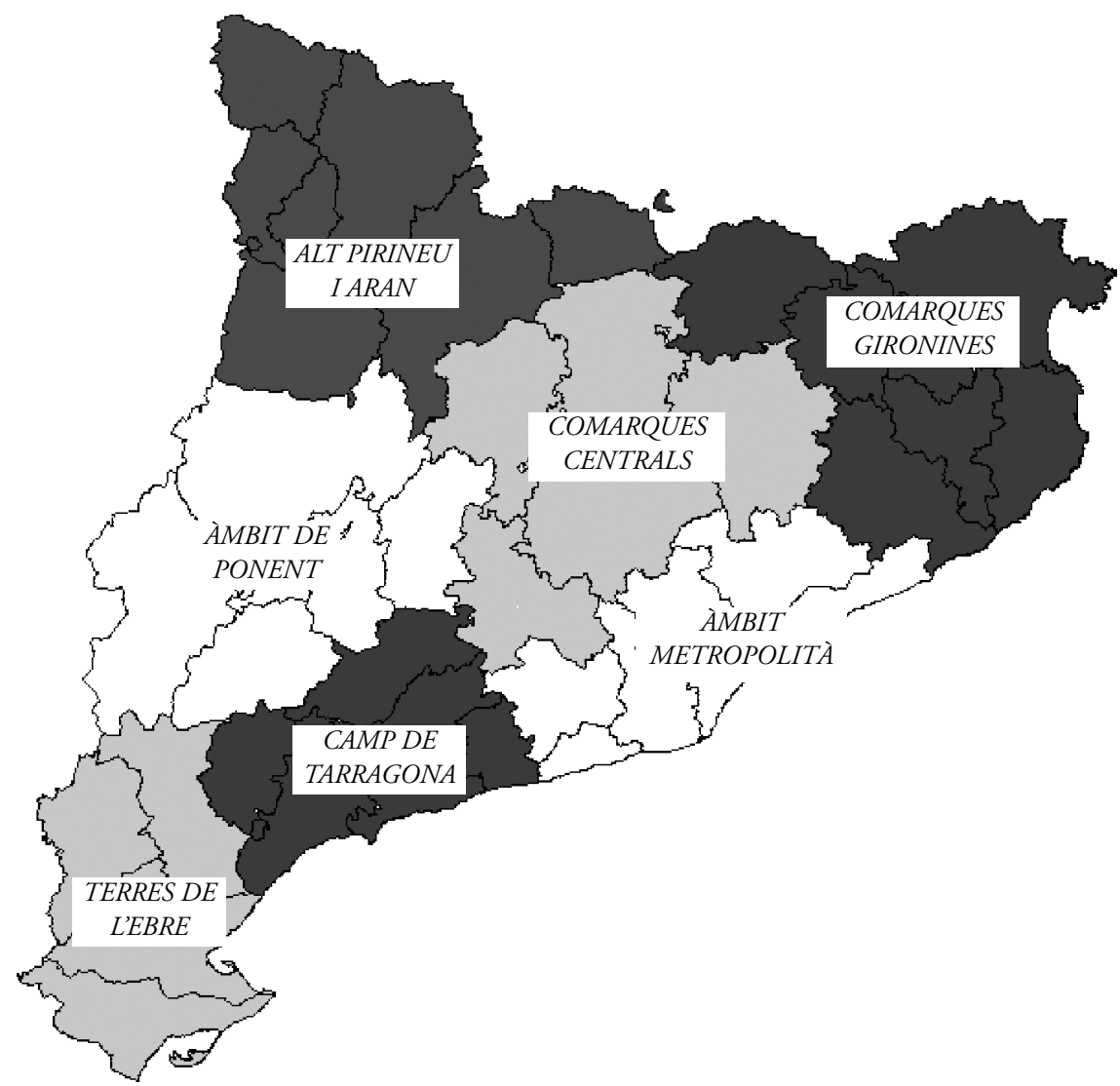

Figura 1. Ámbitos territoriales existentes en Catalunya. Fuente: elaboración propia.

rrente de tipo anidado en la segunda fase. En la figura 2 se representa esquemáticamente la articulación de técnicas que ello ha supuesto. En la primera fase del trabajo de campo, se desarrolló un análisis de los datos estadísticos existentes y un conjunto de discusiones de grupo con expertos de carácter exploratorio. El objetivo del análisis cuantitativo fue contextualizar y situar comparativamente los diferentes ámbitos territoriales y comarcas en las que iba a desarrollarse la segunda fase del diseño, y el de los grupos con expertos, determinar (junto con el conocimiento teórico previo) los temas y los ejes de discusión más relevantes para cada territorio. En la segunda fase se realizaron los grupos de discusión con jóvenes (que fueron analizados mediante un aná- 


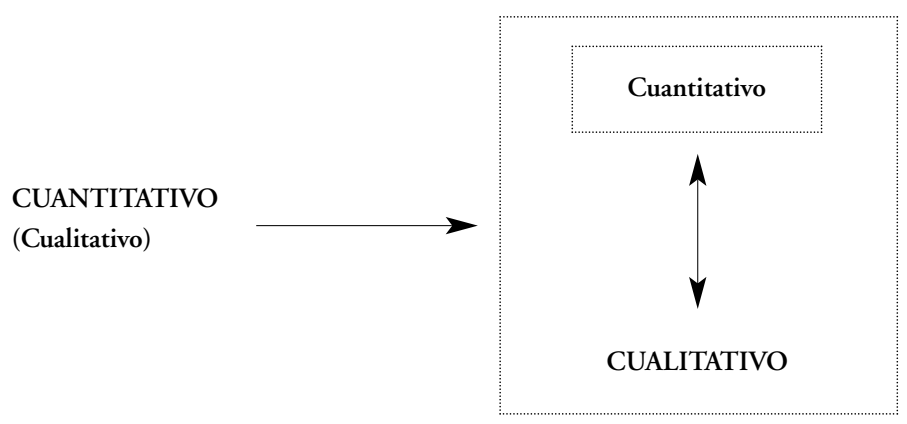

Figura 2. Diseño y articulación de técnicas desarrolladas en la investigación. Fuente: elaboración propia.

lisis temático cualitativo) y se desarrolló un análisis bivariado y trivariado de los datos estadísticos con que contábamos 5 .

Es importante señalar que el análisis estadístico que se realiza en el artículo se fundamenta únicamente en los datos del censo de 2001, que es la base de datos más reciente que no presenta problemas de representatividad a nivel comarcal y de ámbito territorial. Es cierto que la realidad social catalana ha cambiado en algunos aspectos desde la fecha del censo, particularmente por el impacto de la inmigración, pero no existen indicios que lleven a pensar en modificaciones sustanciales de las características socioeconómicas de los contextos territoriales, que es aquello que realmente se analiza en el artículo.

También es relevante señalar que las reflexiones del artículo harán referencia a unas categorías de edad concretas (población entre un límite inferior y superior de edad) que no se corresponden con una definición estrictamente sociológica de «juventud». Abordar el concepto desde una definición estrictamente sociológica exigiría una operativización del concepto que es imposible de realizar considerando sólo la dimensión cronológica (de edad) de la población, por muy flexibles que sean los límites de edad considerados (véase Brunet y Pastor, 2002). No obstante, ello no implica que no pueda hablarse de un concepto de joven en un sentido cronológico (y, por lo tanto, no exactamente sociológico), especialmente útil cuando deben llevarse a cabo análisis con datos de carácter estadístico.

5. Aunque en las dos fases del diseño secuencial se utilizaron datos cuantitativos y cualitativos, en la primera no se produjo un análisis combinado o integrado de dichos datos, ya que unos y otros fueron utilizados con objetivos distintos. En la segunda fase sí se produjo este análisis integrado, es por ello que sólo se ha considerado como diseño concurrente anidado a esta segunda fase. 


\section{Diferencias en la emancipación familiar juvenil por grupos de edad y ámbitos territoriales}

Diversas investigaciones han mostrado, sin lugar a dudas, que la edad de emancipación familiar se está atrasando desde la década de los ochenta, tanto en Catalunya (López, 2005; Miret, 2004a, 2004c) como en el Estado español (CES, 2002, 2006; Jurado, 2003, 2007; Requena, 2006). Este hecho ha tenido como consecuencia más directa la disminución del porcentaje de población en las edades consideradas jóvenes que vive en una vivienda diferente del hogar de origen, aun cuando parecería que se ha dado una cierta estabilización de esta cifra en los últimos diez años (CES, 2006; López, 2005; Requena, 2006) o incluso un ligero aumento en Catalunya — según indican las primeras explotaciones de la Encuesta de Condiciones de Vida y Hábitos de la Población de 2006 (Miralles y Donat, 2007).

Este escaso volumen de jóvenes emancipados familiarmente queda claramente reflejado en los datos que muestra la tabla 1 . Así, entre los 15 y los 34 años sólo el $37,1 \%$ de la juventud vive en un hogar propio ${ }^{6}$. La misma tabla también muestra que el porcentaje de jóvenes emancipados crece con la franja de edad, situándose en el 73\% en los 30-34 años. Este proceso emancipador, no obstante, no termina a los 35 años; tal como ha demostrado Miret (2004b, 2005), para una pequeña parte de la población, se extiende aproximadamente hasta los 40 años, hecho que pone de manifiesto hasta qué punto se está alargando la «juventud» de la población catalana.

Tabla 1. Emancipación familiar por grupos de edad. Catalunya, 2001. Absolutos y porcentajes

\begin{tabular}{lccc}
\hline & \multicolumn{2}{l}{ Jóvenes emancipados } & \\
\cline { 2 - 3 } & Absolutos & Porcentajes & Total jóvenes \\
\hline 15-19 años & 7.868 & 2,3 & 344.485 \\
20-24 años & 68.175 & 14,3 & 476.720 \\
25-29 años & 245.550 & 44,2 & 555.451 \\
30-34 años & 384.553 & 73,0 & 526.588 \\
\hline Total & 706.146 & 37,1 & $\mathbf{1 . 9 0 3 . 2 4 4}$ \\
\hline
\end{tabular}

Fuente: censo de 2001. Institut d'Estadística de Catalunya. Datos elaborados para el estudio La realitat juvenil a Catalunya.

6. Como se ha apuntado anteriormente, todas las tablas que se presentan en el artículo usan datos provenientes del censo de 2001. A pesar de las críticas que, en ocasiones, ha recibido esta base de datos, los porcentajes de emancipación familiar no difieren de los correspondientes a otras fuentes con fecha de trabajo de campo comparable (véase López, 2005: tabla 4). 
Otro dato de relevancia en relación con el proceso de emancipación juvenil en Catalunya es la ya comentada variabilidad territorial que muestran los datos. En la tabla 2, se muestran las cifras absolutas y los porcentajes de emancipados (intensidad) para los diferentes ámbitos territoriales catalanes, distinguiendo entre el grupo de edad de 15 a 24 años y el de 25 a 34 años. Como se puede comprobar, ninguno de los ámbitos se aparta de la tendencia general indicada para el conjunto de Catalunya, pero sí es posible detectar ciertas diferencias en los ritmos de emancipación. Algunos ámbitos tienen un porcentaje de emancipación relativamente elevado en los grupos de edad más jóvenes (15-24 años); éste es el caso de Comarques Gironines, Alt Pirineu i Aran y Camp de Tarragona, que superan el 10\% y destacan por su porcentaje de emancipados por encima del resto. En la situación contraria se encuentran el Àmbit Metropolità y el Àmbit de Ponent, que destacan por sus bajos porcentajes de emancipados en la franja de 15 a 24 años. No obstante, es la franja entre 25 y 34 años la que muestra diferencias más relevantes entre ámbitos territoriales. En esta franja de edad, la mayoría de ámbitos se sitúan con unos porcentajes de emancipados que rondan el $60 \%$. Se alejan de esta tendencia los casos de Terres de l'Ebre (56,2\%), y todavía más los ámbitos de Ponent y del Alt Pirineu i Aran, que se sitúan, respectivamente, en el $52,5 \%$ y en el $52,9 \%$.

La variabilidad territorial que pone de manifiesto la tabla 2 en el grupo de 25 a 34 años es la que se analiza, principalmente, en los siguientes apartados, aunque en algunos casos también se hace referencia al grupo de 15 a 24 años. Tal como se ha comprobado en la tabla 1, es entre los 25 y los 34 años que la juventud catalana se emancipa mayoritariamente. Así, desarrollaremos un análisis focalizado en el nivel territorial y en el grupo de edad más relevante en los procesos de emancipación, dejando de lado expresamente un tipo de trata-

Tabla 2. Emancipación familiar de la población entre 15 y 24 años y entre 25 y 34 años por ámbitos territoriales y para el conjunto de Catalunya, 2001

\begin{tabular}{lccccl}
\hline & $\mathbf{1 5} \mathbf{1 5}$ años & & & 25-34 años & \\
\cline { 2 - 3 } \cline { 5 - 6 } \cline { 5 - 6 } & Absolutos & Porcentajes & & Absolutos & Porcentajes \\
\hline Àmbit Metropolità & 49.637 & 8,8 & & 449.250 & 58,3 \\
Comarques Gironines & 8.928 & 12,5 & & 53.816 & 60,0 \\
Camp de Tarragona & 6.410 & 10,5 & & 45.466 & 60,1 \\
Terres de l'Ebre & 1.873 & 9,1 & 13.194 & 56,2 \\
Àmbit de Ponent & 3.265 & 8,7 & 24.334 & 52,5 \\
Comarques Centrals & 5.129 & 9,2 & & 38.896 & 60,0 \\
Alt Pirineu i Aran & 801 & 11,3 & 5.147 & 52,9 \\
\hline Total Catalunya & $\mathbf{7 6 . 0 4 3}$ & $\mathbf{9 , 3}$ & $\mathbf{6 3 0 . 1 0 3}$ & $\mathbf{5 8 , 2}$ \\
\hline
\end{tabular}

Fuente: censo de 2001. Institut d'Estadística de Catalunya. Datos elaborados para el estudio La realitat juvenil a Catalunya. 
miento más concentrado en las características individuales de los sujetos. De hecho, cuando hablamos de la variabilidad territorial nos estamos refiriendo fundamentalmente a la variabilidad en las características socioeconómicas de cada ámbito territorial, es decir, de los efectos que las condiciones sociales y económicas de cada territorio ejercen sobre el desarrollo de la vida de los jóvenes. Son estos los factores que se van a considerar cuando revisemos separadamente los argumentos que, desde los diferentes modelos teóricos, se usan en la explicación del retraso emancipatorio ${ }^{7}$. En los apartados siguientes, analizaremos por separado estas diferentes argumentaciones teóricas, aplicándolas comparativamente a las diferencias territoriales apuntadas ${ }^{8}$. Si entendemos que los porcentajes observados reflejan intensidades diversas en la tendencia general hacia la demora de la emancipación familiar, resulta interesante comprobar en qué medida los argumentos más generales pueden ser también válidos para explicar las diferencias territoriales.

\section{El peso de los factores económicos y laborales}

Tal y como apunta Gaviria (2007: XIX), el análisis de la emancipación juvenil se realiza, en la mayoría de casos, y casi exclusivamente «de forma cuantitativa, como resultado de factores materiales». El razonamiento seguido es que la emancipación familiar de los jóvenes es consecuencia de la disponibilidad de recursos económicos para afrontar el pago de una vivienda. De este modo, se combinan en la explicación dos factores diferentes: por un lado, el precio de la vivienda y, por el otro, los ingresos derivados de la situación laboral de la joven o el joven. Estos dos argumentos se analizan a continuación por separado, a la luz de los datos ya comentados de la tabla 2 .

Que el incremento de precios de la vivienda dificulta la emancipación juvenil ha sido claramente demostrado, tanto para el caso catalán (DEP y Trilla, 2004: 19-24; López, 2005: 151-154), como para el caso español (CES, 2002: 91-99), midiendo el esfuerzo económico que se debe realizar en un hogar formado por personas jóvenes para pagar una «hipoteca tipo». El espectacular incremento de precios de los últimos años ha tenido consecuencias evidentes sobre este esfuerzo económico, en muchos casos imposibilitando claramente el acceso. Sin embargo, aunque el incremento de precios de la vivienda permite explicar el retraso global de la emancipación juvenil en

7. Véase Leal Maldonado (1997), Gil Calvo (2002) o López (2005) para una revisión crítica.

8. Los razonamientos harán referencia indistintamente a personas de ambos sexos. Como es sabido, el porcentaje de mujeres emancipadas se sitúa siempre por encima del de los hombres, pero no hay ningún ámbito territorial en que una emancipación relativamente alta de hombres no se corresponda con una emancipación también relativamente alta de mujeres (y lo mismo pasa con los ámbitos con emancipación relativamente baja). Esta situación es lógica si pensamos que la forma absolutamente mayoritaria de emancipación continúa siendo en pareja (Casal y otros, 2003; Miret, 2005) y que no hay diferencias territoriales con respecto a este comportamiento. 
Catalunya (y en el Estado español), no parece ser un buen factor explicativo de las diferentes intensidades de emancipación detectadas a nivel territorial. Siguiendo este razonamiento, las comarcas que presentan un porcentaje más bajo de emancipación deberían ser aquéllas en que el esfuerzo económico que realizan los jóvenes es más importante. Pero los análisis realizados considerando diferentes áreas territoriales (DEP y Trilla, 2004; López, 2005) no parecen sostener esta argumentación: el mayor esfuerzo económico lo deben realizar los jóvenes que residen en la conurbación de Barcelona, mientras que los porcentajes más bajos de emancipación corresponden al Alt Pirineu i Aran y al Àmbit de Ponent.

Con todo, la situación de los jóvenes en el mercado laboral permite explicar alguna de las diferencias. Así, resulta fácil atribuir a la estructura económica sectorial las disparidades en los porcentajes en el grupo de 15 a 24 años. Si pensamos que en estas edades los jóvenes que se emancipan familiarmente son fundamentalmente los que tienen escasa formación ${ }^{9}$, podemos relacionar los ámbitos con unos porcentajes más elevados (Comarques Gironines, Camp de Tarragona y Alt Pirineu i Aran) con los sectores (construcción y servicios turísticos) en que mayoritariamente se insertan laboralmente este tipo de jóvenes. Una estructura sectorial particular y diferente de la del resto de ámbitos territoriales podría ser el causante de los niveles de emancipación relativamente más elevados en estas edades.

Resulta muy difícil, en cambio, atribuir a factores económicos o laborales las diferencias observadas en los porcentajes de la franja de 25 a 34 años. Ni entre los ámbitos con un nivel de emancipación relativamente más alto ni entre los que tienen un nivel relativamente más bajo existe una estructura económica análoga. Por otro lado, aquello que puede resultar más sorprendente es el hecho de que los dos ámbitos territoriales con los menores porcentajes de emancipación sean los que tienen las tasas de paro más bajas de toda Catalunya en la franja de 25 a 34 años (6,0\% para el Alt Pirineu i Aran y 6,3\% para el Àmbit de Ponent, según los datos del censo de 2001, frente a un 8,8\% para la media catalana). Esta falta de relación parece dar la razón a aquellas voces (por ejemplo: Serracant, 2001; CES, 2002, o Jurado, 2007) que defienden que es la precariedad y no el paro la que en los últimos años está dificultando la emancipación familiar juvenil. Aun así, las diferencias en los porcentajes de contratación eventual (que ha sido el indicador tomado para operativizar la precariedad) tampoco se pueden relacionar con las diferencias territoriales en el porcentaje de emancipados: la tabla 3 muestra que la eventualidad más alta de las Terres de l'Ebre o del Camp de Tarragona no coincide con los porcentajes de emancipación más bajos observables en la tabla 2; de hecho, los ámbitos con la emancipación más baja son, junto con las Comarques Centrals, los que tienen las tasas de eventualidad también más bajas.

9. Según los datos del censo de 2001, un 19,7\% de los jóvenes de entre 20 y 24 años residentes en Catalunya con un nivel educativo máximo de ESO o EGB ya estaban emancipados familiarmente, cuando la media del resto de jóvenes era del 10,7\%. 
Tabla 3. Población ocupada entre 25 y 34 años con un contrato eventual por nivel de estudios y por ámbitos territoriales. Catalunya, 2001. Porcentajes

\begin{tabular}{lllll}
\hline & $\begin{array}{l}\text { Primarios } \\
\text { o inferiores }\end{array}$ & Secundarios & Universitarios & $\begin{array}{l}\text { Total } \\
\text { estudios }\end{array}$ \\
\hline Àmbit Metropolità & 27,3 & 19,7 & 23,7 & 23,4 \\
Comarques Gironines & 24,8 & 17,6 & 25,0 & 23,5 \\
Camp de Tarragona & 31,3 & 22,2 & 26,9 & 27,1 \\
Terres de l'Ebre & 34,7 & 25,8 & 34,8 & 31,9 \\
Àmbit de Ponent & 25,9 & 18,0 & 27,2 & 22,6 \\
Comarques Centrals & 24,0 & 16,2 & 23,3 & 21,1 \\
Alt Pirineu i Aran & 23,9 & 19,8 & 24,1 & 22,3 \\
\hline
\end{tabular}

Fuente: censo de 2001. Institut d'Estadística de Catalunya. Datos elaborados para el estudio La realitat juvenil a Catalunya.

Los datos que muestra la tabla 3 permiten comprobar, asimismo, que la precariedad tiene tanta incidencia entre la población joven con estudios primarios o inferiores (EGB o ESO como nivel máximo de instrucción) como entre aquélla con estudios universitarios. Dejando de lado las implicaciones que estos datos pueden tener en términos de funcionamiento del mercado laboral y los vínculos entre titulación e inserción para la población joven ${ }^{10}$, es interesante destacar que si fuese la precariedad un factor determinante de la baja emancipación, este fenómeno no se localizaría en las comarcas con una alta presencia de jóvenes con estudios universitarios (como así se puede comprobar más adelante en la tabla 4), sino que también lo haría en las que tienen una elevada presencia de jóvenes con estudios primarios o inferiores, hecho que no sucede si comparamos los datos de la tabla 2 con los de la tabla 4 . En conclusión, los datos no señalan una pauta común entre la distribución de la precariedad y los porcentajes territoriales de emancipación, ni siquiera para un determinado nivel de estudios.

\section{La prolongación de los estudios como factor explicativo}

La ya mencionada tabla 4 es útil también para analizar otro de los factores a los que se suele atribuir el retraso en la emancipación juvenil: el alargamiento del periodo de formación. Leal Maldonado (1997) defiende la existencia de una relación clara entre prolongación de los estudios y emancipación tardía, aunque, al mismo tiempo, afirma que no están del todo claros cuáles son los factores causantes de esta asociación. El mismo autor señala que los argumentos que atribuyen la prolongación de los estudios a las exigencias del mercado labo- 
ral pierden validez hoy en día, en que la formación se convierte en un valor por sí mismo (1997: 119). De esta manera, no siempre se puede atribuir el alargamiento del periodo de formación a las estrategias individuales que buscan la mejora de las posibilidades de inserción en un mercado laboral que supuestamente exige niveles más altos de instrucción. Por otro lado, Miret (2004b, 2004c, 2006) también detecta la existencia de asociación entre un nivel de instrucción alto y el hecho de no estar emancipado. Este autor argumenta (2004c) que es el retraso de la emancipación el factor causante del alargamiento de los estudios, y no al revés. El razonamiento nos remite otra vez a los modelos explicativos que atribuyen las dificultades de emancipación familiar a una mala situación en el mercado laboral, de modo que el alargamiento de los estudios sería una estrategia para mejorar las posibilidades de una buena inserción entre aquéllos que todavía no se habrían emancipado (CES, 2002).

Los datos que presenta la tabla 4 son coherentes con la asociación señalada entre alargamiento de los estudios y emancipación tardía, aun cuando sólo encajan parcialmente con los razonamientos que atribuyen esta asociación a factores vinculados con el mercado de trabajo. Como se puede observar, el Àmbit Metropolità es el que presenta un mayor porcentaje de jóvenes con estudios universitarios, seguido de los ámbitos de Ponent y de Alt Pirineu i Aran. Es muy posible que la elevada oferta formativa presente en el primer ámbito, junto con las buenas comunicaciones internas, favorezca un alargamiento de los estudios por parte de los jóvenes que involuntariamente permanecen en el hogar familiar. Sin embargo, los elevados porcentajes de jóvenes que realizan estudios universitarios en el ámbito de Ponent y en el de Alt Pirineu i Aran son difíciles de atribuir (como mínimo, sin considerar conjuntamente otros elementos) a las dificultades materiales de emancipación, puesto que ya se ha comprobado que son los territorios donde objetivamente estas dificul-

Tabla 4. Población entre 25 y 34 años con nivel de estudios primarios o inferiores y universitarios por sexo y por ámbitos territoriales. Catalunya, 2001. Porcentajes

\begin{tabular}{lllllllll}
\hline & \multicolumn{3}{l}{ Primarios o inferiores } & & \multicolumn{3}{l}{ Universitarios } \\
\cline { 2 - 3 } \cline { 7 - 9 } & Hombres & Mujeres & Total & & Hombres & Mujeres & Total \\
\hline Àmbit Metropolità & 41,4 & 33,8 & 37,7 & & 22,1 & 30,2 & 26,1 \\
Comarques Gironines & 55,0 & 43,3 & 49,3 & & 15,4 & 25,8 & 20,5 \\
Camp de Tarragona & 50,1 & 40,4 & 45,4 & & 16,9 & 25,3 & 21,0 \\
Terres de l'Ebre & 57,4 & 46,8 & 52,4 & & 13,1 & 21,7 & 17,1 \\
Àmbit de Ponent & 47,4 & 34,5 & 41,2 & & 18,7 & 33,0 & 25,6 \\
Comarques Centrals & 50,1 & 39,0 & 44,8 & & 16,7 & 27,1 & 21,7 \\
Alt Pirineu i Aran & 40,9 & 29,9 & 35,5 & & 18,1 & 32,5 & 25,1 \\
\hline
\end{tabular}

Fuente: censo de 2001. Institut d'Estadística de Catalunya. Datos elaborados para el estudio La realitat juvenil a Catalunya. 
tades deberían ser menores. Además, puede añadirse que la oferta formativa de estos ámbitos, especialmente en el caso del Alt Pirineu i Aran, no favorece precisamente una estrategia que opte por el alargamiento del periodo de formación como mal menor ante la imposibilidad de una buena inserción laboral.

Así pues, es necesario ir más allá de los elementos relacionados con el mercado laboral para terminar de identificar todos los factores que inciden en la prolongación de los estudios. El análisis detallado de los datos de la tabla 4 diferenciados por sexo permite avanzar en esta dirección. Como se puede comprobar, las elevadas proporciones de jóvenes con titulación universitaria de los ámbitos de Ponent y de Alt Pirineu i Aran son fruto, fundamentalmente, de la elevada proporción de mujeres con este nivel de instrucción (de hecho son, de toda Catalunya, los dos ámbitos donde mayor diferencia existe entre la proporción de universitarios y la de universitarias: más de 14 puntos). Este hecho indica que una parte considerable de los hombres jóvenes de estos ámbitos se inserta profesionalmente sin disponer de una titulación universitaria; son las mujeres jóvenes las que alargan sus itinerarios formativos (y lo hacen más que nadie en Catalunya). Por lo tanto, los datos parecen indicar que, en ciertos contextos de inserción laboral favorable (como el de los dos ámbitos a los que nos venimos refiriendo), el alargamiento de los estudios se da con mucha mayor intensidad entre las mujeres.

¿Cuáles pueden ser las causas de estos porcentajes especialmente altos de tituladas universitarias precisamente en los dos ámbitos territoriales en que menor porcentaje de emancipados y emancipadas ${ }^{11}$ se observan (y mejores parecen ser las condiciones objetivas para la emancipación)? La singularidad de los ámbitos de Ponent y Alt Pirineu i Aran sugiere la existencia de unos vínculos más complejos que los apuntados hasta ahora en la relación entre la prolongación de los estudios y la emancipación.

En este sentido, posiblemente deban contemplarse factores difíciles de medir estadísticamente (como los apuntados por Leal Maldonado, 1997) que van más allá del valor instrumental de la formación, o como el papel que en la emancipación de los hijos e hijas juegan las estrategias familiares. Ello implica tomar en cuenta las explicaciones fundadas en los aspectos culturales y en las estrategias familiares, que serán abordadas en los dos siguientes puntos. Se trata, de hecho, de dos modelos explicativos diferentes que comparten, por un lado, el hecho de situar las causas de las variaciones del volumen de emancipación en el ámbito familiar y, por otro, la necesidad de utilizar información cualitativa para su contraste.

11. Como se ha señalado en la nota 6 , no hay ningún ámbito territorial en que el porcentaje de emancipados para cada sexo tomado por separado rompa la dinámica observada considerando los dos sexos conjuntamente. Esto significa, para los dos ámbitos a que nos referimos, que coincide un porcentaje comparativamente alto de universitarias con un porcentaje comparativamente bajo tanto de hombres emancipados como de mujeres emancipadas. 


\section{Las estrategias familiares y la clase social de origen}

En el caso de las explicaciones basadas en las estrategias familiares (Carabaña, 1997; Gil Calvo, 2002; Gentile, 2006), el argumento apunta a una responsabilización creciente de las familias en la emancipación de los hijos e hijas, de modo que financiarían tanto la formación considerada necesaria por una buena inserción laboral, como una parte — que puede ser muy considerable— de los costes de la vivienda.

Para analizar correctamente este modelo explicativo, resulta necesario, tal y como hace Carabaña (1997), introducir como factor adicional de relevancia la clase social de las familias. Según este autor, son las familias de clase media las que tienen como estrategia para mejorar las posibilidades de inserción profesional de los hijos e hijas la utilización de sus recursos económicos en la provisión de credenciales educativas. No deja de ser sorprendente que, a pesar de la importancia que se otorga a la clase social como factor explicativo en muchos de los ámbitos de investigación de la sociología, éste tenga tan poca presencia en los trabajos empíricos que tratan la emancipación juvenil ${ }^{12}$. Una de las razones puede estribar en las dificultades de operativización del concepto, que aquí se ha aproximado mediante la utilización de la categoría socioeconómica ${ }^{13}$, cuya distribución territorial se presenta en la tabla 5.

Tabla 5. Categoría socioeconómica de la población de 25 a 34 años y de más de 34 años por ámbitos territoriales. Catalunya, 2001. Porcentajes

\begin{tabular}{llllllll}
\hline & \multicolumn{3}{c}{ Población de 25 a 34 años } & & \multicolumn{3}{c}{ Población de más de 34 años } \\
\cline { 2 - 3 } \cline { 7 - 8 } & Baja & Media & Alta & & Baja & Media & Alta \\
\hline Àmbit Metropolità & 62,0 & 31,4 & 6,6 & & 56,8 & 31,3 & 11,9 \\
Comarques Gironines & 65,2 & 27,7 & 7,1 & & 58,4 & 27,9 & 13,7 \\
Camp de Tarragona & 65,5 & 27,5 & 6,9 & & 57,4 & 30,0 & 12,6 \\
Terres de l'Ebre & 65,5 & 27,7 & 6,9 & & 54,3 & 33,5 & 12,2 \\
Àmbit de Ponent & 59,1 & 33,2 & 7,6 & & 50,7 & 35,6 & 13,7 \\
Comarques Centrals & 64,4 & 28,9 & 6,7 & & 59,4 & 28,6 & 12,0 \\
Alt Pirineu i Aran & 58,0 & 32,2 & 9,8 & & 47,7 & 35,8 & 16,5 \\
\hline
\end{tabular}

Fuente: censo de 2001. Institut d'Estadística de Catalunya. Datos elaborados para el estudio La realitat juvenil a Catalunya.

12. De hecho, Gil Calvo, aunque defiende la explicación basada en las estrategias familiares (2002: 16-17), no hace ninguna distinción en función de la clase social.

13. Los datos del censo no recogen ningún tipo de información que permita hacer operativo el concepto de clase social de forma directa, es por ello por lo que usamos la categoría socioeconómica, que se puede construir del mismo modo que se hace a la encuesta de población activa desde el año 1994. Los porcentajes de la tabla 5 son el resultado de agrupar los diecinueve valores de la variable original del censo en tres categorías: baja, mediana y alta (véase el anexo 1 de López, Valls, Verd y Vidal, 2006). 
Considerando la distribución que la categoría socioeconómica presenta, tanto entre la población de entre 25 y 34 años como en la de más de 34 años, resulta destacable que los ámbitos de Ponent y de Alt Pirineu i Aran sean los que tienen la proporción más elevada de personas con categoría socioeconómica media o alta, concretamente un $49,3 \%$ y un $52,3 \%$, respectivamente, para la población de más de 34 años, y un 40,8\% y un 42,0\%, respectivamente, para la población de 25 a 34 años. Como se comprueba, son exactamente los dos ámbitos en que son menores los porcentajes de jóvenes emancipados y emancipadas de entre 25 y 34 años. Resulta verosímil pensar, por lo tanto, que la estrategia de inversión familiar educativa en los ámbitos que analizamos - con un contexto territorial donde los hombres jóvenes ya tienen comparativamente una buena inserción laboral sin necesidad de prolongar su itinerario formativo - se da sobre todo en el caso de las mujeres jóvenes (tal y como ponían de manifiesto los datos de la tabla 4). Es interesante destacar que la precariedad de las chicas de 25 a 34 años del Alt Pirineu i Aran y del Âmbit de Ponent se sitúa en la franja baja del conjunto de ámbitos territoriales, junto con la del Àmbit Metropolità y la de las Comarques Centrals. No obstante, está siempre unos cinco puntos por encima de la de los chicos de los mismos ámbitos, hecho que podría justificar esta inversión "extra» en formación. De hecho, resulta relevante que precisamente en los grupos de discusión realizados con chicas de clase media, la necesidad de inversión «extra» en formación como vía para conseguir una situación laboral equiparable a sus coetáneos masculinos, fuese mencionada reiteradamente. En este caso estaríamos, por lo tanto, ante una situación que podría explicarse por la combinación de una determinada situación (económica) familiar de partida y una búsqueda de mejora en las expectativas de inserción en el mercado laboral.

Con todo, es conveniente no olvidar que, retomando el argumento de Leal Maldonado (1997), los estudios universitarios combinan en muchos casos el valor instrumental y el expresivo, y que, además, son igualmente generadores de estatus (tal y como se muestra en las investigaciones de Planas, 1993, o Verd, 2002, entre otras). Igualmente, los trabajos de Bourdieu (1988, 1991) muestran que, para las familias de clase media, la inversión educativa no está necesariamente orientada a la inserción laboral, sino también a mejorar o mantener el estatus familiar de partida. Estas estrategias de reproducción cultural y de estatus vinculadas a la clase social deben explorarse mediante el uso de la información de carácter cualitativo obtenida en los grupos de discusión, tarea a la que se dedica el siguiente apartado. A su vez, el análisis de esta información permitirá realizar una aproximación a los factores que el modelo explicativo culturalista considera más relevantes.

\section{Los factores culturales y las expectativas en la emancipación}

En las explicaciones culturalistas de la emancipación familiar (Gaviria, 2002, 2007; Gil Calvo, 2002: 13-16, Leal Maldonado, 1997: 118), el argumento principal apunta al peso creciente entre la juventud de las preferencias por una 
emancipación más cómoda - lo cual suele implicar también que sea más tardíapropiciada por unos márgenes elevados de libertad en el seno de las familias y una buena relación de convivencia con los progenitores. Las informaciones provenientes de los grupos de discusión realizados con jóvenes de clase media no pertenecientes al Àmbit Metropolità aportan elementos ${ }^{14}$ que hacen pensar en un triple vínculo entre clase social, prolongación de los estudios y emancipación tardía. Las expectativas expresadas por estos jóvenes no sólo van en la dirección de alargar su periodo de formación hasta la universidad, sino que también ponen de manifiesto que el tipo de emancipación en la que se piensa se acerca mucho a un modelo tradicional en qué sólo se deja de convivir con los padres para ir a vivir con la pareja o casarse. Frente a las dificultades de emancipación, se prefiere continuar residiendo en casa de los padres, aunque ello implique tener menor libertad o intimidad. En la práctica, en muy pocas ocasiones, los jóvenes se plantean un tipo de emancipación compartiendo piso o en solitario, posibilidad que sólo conciben aquéllos que ya conocen la experiencia de compartir alojamiento por el hecho de haber ido a estudiar fuera de sus zonas de origen. En este sentido, la información cualitativa coincide con otras investigaciones (Casal y otros, 2003, 2004; Miret, Martorell y Segon, 2002; Gaviria, 2007) que apuntan hacia una tendencia a permanecer en casa de los padres hasta el momento de la emancipación con la pareja, situación reforzada - o cuando menos sostenida - por el familismo que caracteriza buena parte de la sociedad catalana y española. Por otro lado, es interesante destacar que precisamente en estos grupos de discusión con un perfil de clase media no metropolitana, los jóvenes piensan en una emancipación familiar basada en la compra de vivienda, que, idealmente, sería una casa unifamiliar.

Gaviria (2007), si bien no estudia específicamente el caso catalán, explica perfectamente la lógica subyacente entre estos jóvenes de clase media no metropolitana. La transición hacia la vida adulta y la propia construcción de la identidad se hace en el seno de la familia, y no como oposición a ella (como ocurre en otros contextos). Ello significa que el modelo de emancipación que se desea por parte de los jóvenes se corresponde de forma substancial con las preferencias de los padres. Dicho de otro modo: la estrategia emancipatoria de los hijos e hijas coincide y se articula con la estrategia sucesoria de los padres (Gil Calvo, 2002: 16). De esta manera, es en la familia donde convergen los factores destacados por las explicaciones culturalistas (expectativas de emancipación y construcción de la identidad juvenil) y los destacados por las explicaciones basadas en las estrategias familiares (responsabilización de la familia ${ }^{15}$ en la reproducción social y económica de la siguiente generación). Lo que no

14. El detalle de estas informaciones puede consultarse en los informes para los ámbitos territoriales no metropolitanos de López, Valls, Verd y Vidal (2006).

15. En los estudios basados en la comparación internacional (Gentile, 2006; Gaviria, 2007), se asocia esta responsabilización, en buena medida, a factores institucionales y, más concretamente, al papel ejercido por las políticas sociales del Estado en esta tutela de la emancipación. 
siempre se señala desde estos dos modelos es que la lógica explicativa que acabamos de mencionar sólo funciona con unas determinadas condiciones materiales de las familias, que, a su vez, están marcadas por las características socioeconómicas de los territorios donde habitan.

En definitiva, parece que un análisis conjunto de la información de carácter cuantitativo y cualitativo permite defender la existencia de relación entre un contexto económico favorable, en que las familias disponen de recursos para pagar el alargamiento del periodo formativo de los hijos e hijas — si hace falta - y una emancipación especialmente tardía, derivada de un modelo de emancipación de carácter tradicional. Una autonomía suficiente de los jóvenes en el contexto familiar fortalecería esta tendencia a permanecer en casa de los padres. Los vínculos de esta combinación de factores con el tipo de vivienda escogida como residencia ya han sido anteriormente destacados, especialmente en el trabajo de Jurado (2003), que pone de manifiesto que el matrimonio - a parte de ser la forma mayoritaria de convivencia por parte de los jóvenes emancipados - está estrechamente vinculado a la adquisición de una vivienda en propiedad. En tanto en cuanto alguno de estos factores no esté presente o lo esté en menor medida, la emancipación no será tan tardía, ya sea porque se renuncia a la emancipación mediante la compra de una vivienda, ya sea porque la familia no asume (no quiere o no puede) la tarea de apoyo económico y reproducción del estatus familiar, ya sea porque la construcción identitaria y el patrón seguido en la emancipación no sigue el modelo tradicional. Esta combinación de factores podría explicar el porqué de intensidades en la emancipación relativamente elevadas en comarcas con una presencia menos significativa de familias de categoría socioeconómica media o alta, o en las de carácter más metropolitano, en qué el modelo tradicional de emancipación está menos extendido.

Para finalizar, y a pesar de todo el razonamiento que hemos seguido en este apartado, es primordial no perder de vista que el retraso en la emancipación es, en buena medida, una situación no deseada. Los datos de carácter estadístico que muestran el esfuerzo económico necesario para la compra de una vivienda llevan a pensar que el incumplimiento de alguna de las expectativas vinculadas a un modelo tradicional de emancipación se resuelve, en el corto plazo, con un retraso considerable en el abandono del hogar familiar. Es importante no olvidar que las condiciones materiales que dificultan la emancipación afectan, aunque sea en diferentes grados, a todo el territorio catalán. Por lo tanto, no siempre las circunstancias reales coinciden con las deseadas, lo cual implica que la emancipación, cuando finalmente se lleve a cabo, se desarrolle en peores condiciones que las esperadas.

\section{Conclusiones}

El artículo ha puesto de manifiesto, en primer lugar, y a partir del análisis de datos censales, las diferencias territoriales existentes en Catalunya en los porcentajes de emancipación juvenil. Si bien los trabajos empíricos existentes han 
tendido a ignorar estas disparidades territoriales — para concentrarse en otras dimensiones de la problemática-, es importante poner de manifiesto que estas diferencias no son más que un reflejo de las condiciones sociales y económicas en qué viven las jóvenes y los jóvenes.

Una vez constatada esta disparidad, se ha procedido a comprobar, utilizando información tanto cuantitativa como cualitativa, cuáles de los factores destacados por los diferentes modelos teóricos que dan cuenta del retraso en la emancipación juvenil permitían explicar estas diferencias territoriales.

De entre estos modelos, el más difundido - y el que se ha revisado en primer lugar - es el que atribuye la intensidad de la emancipación juvenil a factores económicos y laborales, donde el incremento de los precios de la vivienda y la situación de la juventud en el mercado laboral tienen un papel central. El análisis realizado en las páginas precedentes ha mostrado que estos factores no sirven por sí solos para explicar las diferencias territoriales detectadas en los grupos de jóvenes de edad más elevada (de 25 a 34 años), puesto que son precisamente los territorios con porcentajes de paro y precariedad más bajos (y también con mayor proporción de adultos con niveles socioeconómicos altos o medios) los que presentan unos porcentajes de emancipación familiar más reducidos. Ello no implica necesariamente que estos modelos estén equivocados, puesto que han demostrado ser útiles para interpretar globalmente el retraso emancipatorio observado en los últimos veinte años en el conjunto de personas de entre 15 y 34 años. Ahora bien, una vez explicada la tendencia general común a todo el espacio catalán (y español), son claramente insuficientes para explicar las diferencias entre territorios.

El uso combinado de información cuantitativa y cualitativa ha permitido comprobar la influencia de algunos otros factores explicativos, más allá de los estrictamente económicos o laborales. Así, se ha podido comprobar que los porcentajes más bajos de emancipación en los jóvenes de entre 25 y 34 años se dan en los territorios en que coinciden una proporción comparativamente elevada de familias con unas condiciones económicas favorables y un modelo de emancipación de carácter tradicional. Atribuir este atraso al hecho de que los jóvenes y las jóvenes de estos territorios permanezcan más tiempo que otros dentro del sistema educativo es excesivamente reduccionista, y deja de lado las causas últimas del alargamiento de los estudios. Las razones son posiblemente más complejas, y deberían situarse, por una parte, en las estrategias familiares que intentan asegurar una inserción laboral de calidad de los hijos e hijas (y no siempre los estudios superiores son el medio para conseguirlo), y, por otra, en una cultura de clase que, a la vez que otorga a los estudios universitarios un valor de estatus, tiene unos imaginarios muy concretos sobre el tipo de emancipación deseada.

Las consideraciones precedentes no deben entenderse como negación de la incidencia de los factores económicos en la emancipación —especialmente en un contexto como el actual en que el nivel de los precios de la vivienda es extraordinariamente alto-, sino como advertencia ante la tentación de convertir la ausencia de unas determinadas condiciones materiales en único fac- 
tor explicativo del retraso de la emancipación juvenil. Esta disposición de recursos es siempre relativa (respeto a unas expectativas de vivienda marcadas en muy buena parte por una determinada cultura de clase), y se combina con factores vinculados a las estrategias familiares y a los valores sociales y culturales predominantes en un determinado territorio.

Para terminar, es importante mencionar que los aspectos examinados en el artículo son aquéllos que se han extraído de los modelos explicativos considerados más relevantes, y que posiblemente no agotan la multiplicidad de factores que influyen en los niveles de emancipación. El trabajo de análisis realizado no deja de ser una aproximación a una realidad juvenil que es cada día más heterogénea y diversa. Debe destacarse, no obstante, que ha sido el uso combinado de datos cuantitativos y cualitativos lo que ha permitido contrastar la influencia de factores tan diversos como los señalados por los diferentes modelos revisados. El uso de información cualitativa ha permitido operativizar factores que difícilmente se pueden abordar con datos de carácter estadístico, y la información cuantitativa ha permitido contextualizar y "confrontar» la información cualitativa obtenida en los grupos de discusión con las condiciones materiales de existencia de los participantes. Por otro lado, la información cuantitativa ha permitido abordar la diversidad territorial y evaluar la relevancia de los factores cualitativos, evitando caer en la particularidad y la anécdota.

\section{Bibliografía}

BERICAT, Eduardo (1998). La integración de los métodos cuantitativo y cualitativo en la investigación social. Barcelona: Ariel.

Bourdieu, Pierre (1988). La distinción. Criterios y bases sociales del gusto. Madrid: Taurus.

Bourdieu, Pierre (1991). El sentido práctico. Madrid: Taurus.

BRUNET, Ignasi; PASTOR, Imma (2002). «Aproximacions teòriques a la inserció laboral dels joves». Arxius de Ciències Socials, 6: 55-82.

CARABAÑA, Julio (1997). «Educación y estrategias familiares de reproducción». En: Luis GARRIDO MEDINA y Enrique GIL CALVO (ed.). Estrategias familiares. Madrid: Alianza.

Casal, Joaquim; Garcia, Maribel; Merino, Rafael; Miguel, Francesc J. (2003). Enquesta als joves de Catalunya 2002. Avançament de resultats. Barcelona: Secretaria General de Joventut de la Generalitat de Catalunya.

Casal, Joaquim; Garcia, Maribel; Merino, Rafael; Miguel, Francesc J. (2004). Enquesta als joves de Catalunya 2002. Barcelona: Secretaria General de Joventut de la Generalitat de Catalunya.

CES (2002). La emancipación de los jóvenes y la situación de la vivienda en España. Madrid: Consejo Económico y Social.

CES (2006). El papel de la juventud en el sistema productivo español. Madrid: Consejo Económico y Social.

CRESWell, John W. (2003). Research Design. Qualitative, Quantitative, and Mixed Methods Approaches. Thousand Oaks, California: Sage.

Creswell, John W.; Plano Clark, Vicki L. (2007). Designing and conducting Mixed Methods Research. Thousand Oaks, California: Sage. 
DEP Consultoria EstratèGiCA; Trilla, Carme (2004). Els joves catalans i l'habitatge 1999-2003. Barcelona: Secretaria General de Joventut de la Generalitat de Catalunya.

DOMÍNGUEZ, Màrius; CoCO, Andrés (2000). «El pluralisme metodològic com a posicionament de partida. Una primera valoració del seu ús a la recerca social». Revista Catalana de Sociologia, 11: 105-132.

FERnÁNDEZ, Cristina; BATALLA, Josepa; Micó, Joan R. (2005). L'emancipació dels joves d'Andorra. Sant Julià de Lòria: Centre de Recerca Sociològica.

GAVIRIA, Sandra (2002). «Retener a la juventud o invitarla a abandonar la casa familiar: Análisis de España y Francia». Estudios de Juventud, 58: 1-6.

GAVIRIA, Sandra (2007). Juventud y familia en Francia y en España. Madrid: CIS-Siglo XXI.

GENTILE, Alessandro (2006). Una precaria transición a la edad adulta: inestabilidad laboral y límites del régimen familista del Estado del Bienestar. El caso de España. Documento de Trabajo 06-02. Unidad de Políticas Comparadas (UPC). Consejo Superior de Investigaciones Científicas.

GIl CALVO, Enrique (2002). «Emancipación tardía y estrategia familiar (El caso de los hijos que ni se casan ni se van de casa)». Estudios de Juventud, 58: 9-18.

JURADO, Teresa (2003). «La vivienda como determinante de la formación familiar en España desde una perspectiva comparada». Revista Española de Investigaciones Sociológicas, 103: 113-157.

JURADO, Teresa (2007). «La precariedad temporal-salarial y sus efectos sobre la formación familiar». Sociedad y Utopía, 29: 367-404.

LALLEMENT, Michel (2003). «Raison ou trahison? Éléments de réflexion sur les usages de la comparaison en sociologie». En: Michel LaLlement y Jan SPURK (dir.). Stratégies de la comparaison internationale. París: CNRS Editions.

LEAL MADONADO, Jesús (1997). «Emancipación y vivienda». En: Ricard VERGÉs (ed.). La edad de emancipación de los jóvenes. Barcelona: Centre de Cultura Contemporània de Barcelona.

LÓPeZ, Pedro; Valls, Núria; Verd, Joan Miquel; Vidal, Pau (coords.) (2006). La realitat juvenil a Catalunya [en línea]. Informes comarcales, por ámbito territorial y para Catalunya. Observatori Català de la Joventut, Secretaria General de Joventut de la Generalitat de Catalunya. <http://www20.gencat.cat/portal/site/Joventut> [Consulta: 14 de noviembre de 2007]

LÓPEZ, Jofre (2005). «Habitatge». En: SECRETARIA GENERAL DE JoventuT: Informe sobre la joventut al 2005. Barcelona: Secretaria General de Joventut de la Generalitat de Catalunya.

LozAres, Carlos; MarTín, Antonio; López, Pedro (1998). «El tratamiento multiestratégico en la investigación sociológica». Papers, 55: 27-43.

MiRAlLES, Carme; DONAT, Carles (2007). «Característiques de la demanda jove d'habitatges a la Regió Metropolitana de Barcelona». ACE. Arquitectura, Ciudady Entorno, 5: 427-441.

MireT, Pau (2004a). Emancipació domiciliària, laboral i familiar dels joves a Catalunya. Barcelona: Secretaria General de Joventut de la Generalitat de Catalunya.

Miret, Pau (2004b). Una visió de la joventut a Catalunya a la llum de la primera onada del Panel de Desigualtats Socials a Catalunya (PaD) [en línea]. Barcelona: Fundació Jaume Bofill. <http://www.obdesigualtats.cat http://www.obdesigualtats.org/ obd/lstcontenido.php?IDC=64\&IDA=2> [Consulta: 14 de noviembre de 2007]

MiRET, Pau (2004c). “¿Qué relación tiene el aumento de los años de escolaridad no obligatoria en la emancipación de los jóvenes en Cataluña durante la segunda 
mitad del siglo XX?». Papers de Demografia, núm. 249 [en línea]. <www.ced.uab.es/ publicacions/PapersPDF/Text249.pdf> [Consulta: 14 de noviembre de 2007] MiRET, Pau (2005). «Conviure en parella, matrimonis versus cohabitació consensual. Catalunya, 1991, 1996 i 2001». Papers de demografia, núm. 256 [en línea]. <www.ced.uab.es/publicacions/PapersPDF/Text256.pdf> [Consulta: 14 de noviembre de 2007]

MireT, Pau (2006). «Escolarización, mercado de trabajo y emancipación familiar en España: Un análisis longitudinal a escala de Comunidad Autónoma». Papeles de Geografia, 43: 73-92.

Miret, Pau; MARTORELl, Victòria; SEGON, Jordi (2002). «Emancipació juvenil a la ciutat de Barcelona». Papers de Demografia, núm. 204 [en línia]. $<$ www.ced.uab.es/publicacions/PapersPDF/Text204.pdf> [Consulta: 14 de noviembre de 2007]

PLANAS, Jordi (1993). Els usos formatius dels joves i la seva inserció professional. Tesis doctoral. Barcelona: Universitat Autònoma de Barcelona. Departament de Sociologia.

REQUENA, Miguel (2006). «Familia, convivencia y dependencia entre los jóvenes españoles». Panorama Social, 3: 64-77.

Salvadó, Antoni (2005). «Treball». En: SeCretaria General DE Joventut: Informe sobre la joventut catalana al 2005. Barcelona: Secretaria General de Joventut de la Generalitat de Catalunya.

Serracant, Pau (2001). Viure al dia: Condicions d'existència, comportaments i actituds dels joves catalans. Informe sobre la joventut catalana al 2001. Barcelona: Secretaria General de Joventut de la Generalitat de Catalunya.

TASHAKKORI, Abbas; TEDDLIE, Charles (eds.) (2003). Handbook of mixed methods in social and behavioral research. Thousand Oaks, California: Sage.

TRILlA, Carme; LÓPEZ, Jofre (2005). «El acceso de los jóvenes a la vivienda: una cuestión todavía no resuelta». Documentación Social, 138: 191-206.

VERD, Joan Miquel (2002). Itinerario biográfico, recursos formativos y empleo. Una aproximación integrada de carácter teórico y metodológico. Tesis doctoral [en línea]. Barcelona: Universitat Autònoma de Barcelona. Departament de Sociologia. <http://www.tdr.cesca.es/index_tdx_cs.html> [Consulta: 15 de octubre de 2007]

VERGÉS, Ricard (1997). «Introducción: el retraso en la edad de la emancipación». En: Ricard Vergés (ed.). La edad de emancipación de los jóvenes. Barcelona: Centre de Cultura Contemporània de Barcelona. 